\title{
An Efficient Meta Scheduling based Virtual Consolidation for Resource Sharing in Green Cloud
}

\author{
S. Lavanya Prabha and R. Dhivya
}

\begin{abstract}
In modern researchers, cloud parallel data processing has emerging resource that to be one of the problematic application for Infrastructure-as-a-Service (IaaS) clouds. Major Cloud processing companies include starting incorporate frameworks using VM models for parallel data processing in their resource portfolio creation to easy for a client to access these services and to set out their programs. The growing computing requires from multiple requests on the main server has lead to excessive power utilization. The waiting resource in the long-term sustainability of Cloud like infrastructures in provisions of energy cost but also from cloud environmental perspective. The trouble can be addressed to require with high energy consumption resource sharing infrastructures, but in the process of resources are dynamically switch to new infrastructure. Switching is not enough to cost efficient and also need time sharing green consuming. Cloud being consists of several virtual centers like $V M$ 's under the different administrative domain, make a problem more difficult. Thus, for the reduction in energy consumption, this propose address the challenge by effectively distributing compute-intensive parallel applications on the cloud. To propose a Meta-scheduling algorithm, this exploits the heterogeneous nature of Cloud to achieve the reduction in energy consumption as the green cloud. This intent addresses these challenges by proposing a virtual file system specifically optimized for virtual machine image storage. It is based on a lazy transfer scheme coupled with object versioning that handles snapshot ting transparently in a hypervisorindependent fashion, ensuring high portability for different configurations.
\end{abstract}

Keywords--- Cloud Computing, Data Centers, Data Distributions, Virtual Machine or Migration.

\section{INTRODUCTION}

Cisas LOUD is a centralized resource to share the resource that has a facility to consume scalably, scattered computing environments within the boundaries of resource sharing on the Internet, a practice that shares data in cloud computing. In this innovative world of the computing process, clients are collectively required to recognize the underlying principle of trust. Within the cloud resource sharing environment, the

S. Lavanya Prabha, ME (CSE), Paavai Engineering College, Pachal, Namakkal, Tamil Nadu. E-mail:lavanyaprabhapecme@gmail.com

R. Dhivya, ME., Assistant Professor, Department of CSE, Paavai Engineering College, Pachal, Namakkal, Tamil Nadu DOI: 10.9756/BIJDM.8303 virtual sharing data users access the resources with gained power limits that do not exceed that limited within their individual physical worlds as well as the green cloud. Cloud provides the centralized resource sharing facilities via the internet. And it's provided us better and efficient way to access information promptly and also increases storage of capacity for the user in.

Cloud is a middle ware process for data compelling technology. In clouds, clients can dynamically request or allocate their resources using on-demand service without sophisticated exploitation and supervision of resources sharing. To enabling the key technologies in clouds, they include the Virtual machine processing paradigm VM's migrates the distributed file systems virtualization and so forth for request sharing. These procedures highlight the scalability, so clouds can be huge in extent resource provider, and embrace entities can subjectively be unsuccessful and join while maintaining system reliability for a green cloud. Distributed file structure are basic building blocks using Virtual data sharing in cloud applications based on the Virtual machine programming standard. In this type of file systems, the nodes are simultaneously deliver or share the resources and maintain the storage functions dynamically to request file is divided into switched allocated space in dissimilar nodes. So that Virtual machine tasks can be performed in parallel workloads over the dissimilar nodes. For instance, consider a packet count application that counts the number of dissimilar packets and the frequency of each unique packet that merge in a large data file. Dissimilar files are delivered by different resource and merged by reference Packet. Each storage space nodes (or node for short) then estimate the occurrence of each unique data packets by examining and parsing its confined file portion. In such a dispersed file organization, the weight of a node is characteristically proportional to the number of file chunks the node possesses. Because the files in a cloud can be arbitrarily allot, share, verify and nodes can be upgraded, replaced and added in the file organization the file large portion are not disseminated as uniformly as probable among the nodes. Load balance surrounded by storage nodes is a dangerous function in clouds sharing. In a load-balanced cloud, the possessions can be well exploit and provisioned, exploit the performance of extended Virtual machine-based applications. State-of-the-art distributed file systems in clouds rely on innermost nodes to manage the metadata information of the file systems and to stability the loads of storage nodes based on that metadata. The centralized approach simplifies the design and implementation of a distributed file system. 
However, recent experience concludes that when the number of storage nodes, the number of files and the number of accesses to files increase linearly, the central nodes (e.g., the master in Google service provider) become a performance bottleneck to create shared issues.

\section{RELATED WORK}

Deploying unimportant datasets across a few servers are the important task. However, organizing of data across hundreds or thousands of servers becomes considerably additional challenging after a performance standpoint. Substantial quantities of data must be transported to each newly created VM [1].Data distribution methods should focus on reducing the overall transmission time. Dropping the total burden of data relocate around the mitigation that reduces the collision of organized service such a service on be the demand to take time[2].

It must also be considered that a data center environment provides no guarantees of available traffic, which can render any estimate based approaches useless. In this subset, we analyze the different approaches for performing data distribution among the deployed VMs. Cloud computing is a model which enables on request network access to a shared pool computing resources. A cloud atmosphere holds the multiple clients request for resources in a dynamic data sharing with possible restriction [3, 4]. In existing system cloud computing, allocating the resource usually is a challenging job. The cloud does not show the quality of services. The resource provides the limitation to dimension to the virtual infrastructure, data request and waiting state and total job allotment in the cloud provider to use.

In addition to the topology of the virtual cluster, input files, and output folder locations are captured. The configuration parameters, a total number of machines, local directories where the data will be located, data files names, etc. are captured. Finally, the cloud provider credentials, token, and the like [6]. All these data resource provided by virtual configuration, resources additional referred to as arrangement of data parameters. This is the barely client interaction needed to access, the rest of the progression is repeatedly done by the intent framework.

A scheme which can automatically scale its infrastructure resources has intended the system composed of a virtual network of simulated machines capable of live migration across multi-domain physical infrastructure Cloud computing facilities providers bring their resources based on virtualization to satisfy the need of users. In cloud computing, the amount of incomes required can vary preserve application $[8,9]$. Cloud has centralized resource to the providers have to present dissimilar amounts of virtualized possessions as per application. The cloud computing services are conveying over the internet there may be undesirable response latency between the users and the database. Hence, for the best recent service, the provider needs to find a data center and physical machine that has a light workload and is geographically close to the users [10].

\section{A. Problem Identification Factors}

Growing numerals of cloud companies have to development huge quantity of data in a cost-efficient sharing manner. Classic resource legislatures for this business are operators of Internet search engines require centralized servers. The enormous quantity of data they encompass to deal with every day data processing has made conventional database solutions prohibitively expensive. The require for low latency investigation over high-velocity data torrent resource stimulate the need for scattered continuous dataflow systems. Existing stream processing structure uses straightforward practice to scale on extended cloud resources to switch different servers. However, application QoS is also impacted by variability in resource performance exhibited by clouds and hence demands autonomic technique of provisioning extended resources to support data sharing applications on cloud infrastructure. The preceding perception of dynamic dataflow which exploits alternate workload tasks as supplementary control over the dataflow's cost and QoS service. Further, we consecrate an optimization problem to represent consumption and runtime reserve provisioning data that allows us to balance the application's QoS, value, and the resource cost. Problems like processing crawled documents or regenerating a web index are split into several independent subtasks, distributed among the available nodes, and computed in parallel.

- The virtual server is congested and can lead to corrupts file performance of its neighbor VMs.

- Overall utilization of servers in the face of multidimensional resource constraints is low.

- Servers in many existing data centers are often severely underutilized due to over provisioning for the peak demand.

- They condense the number of sharable energetic servers during high load without deliberate switching process in the difficult state.

\section{PROPOSED SOLUTION}

To develop a practical meta-scheduling cloud resource VM allocation algorithm. It is competent of together overload mitigation avoidance, and green computing is reduced. It is appropriate to scenarios with multi-dimensional resource sharing constraints. We provide an imaginary proof that the integer of servers is allotted in and VM is the number of extended resources. The VM generalize the content service provider with maximum priority requested to size estimation to distribute requested resource, the number of servers in use is bounded by times of switching the optimal value. We also establish that the numeral of VM migration is bounded as well. We accomplish widespread delivery data and accomplished time. 


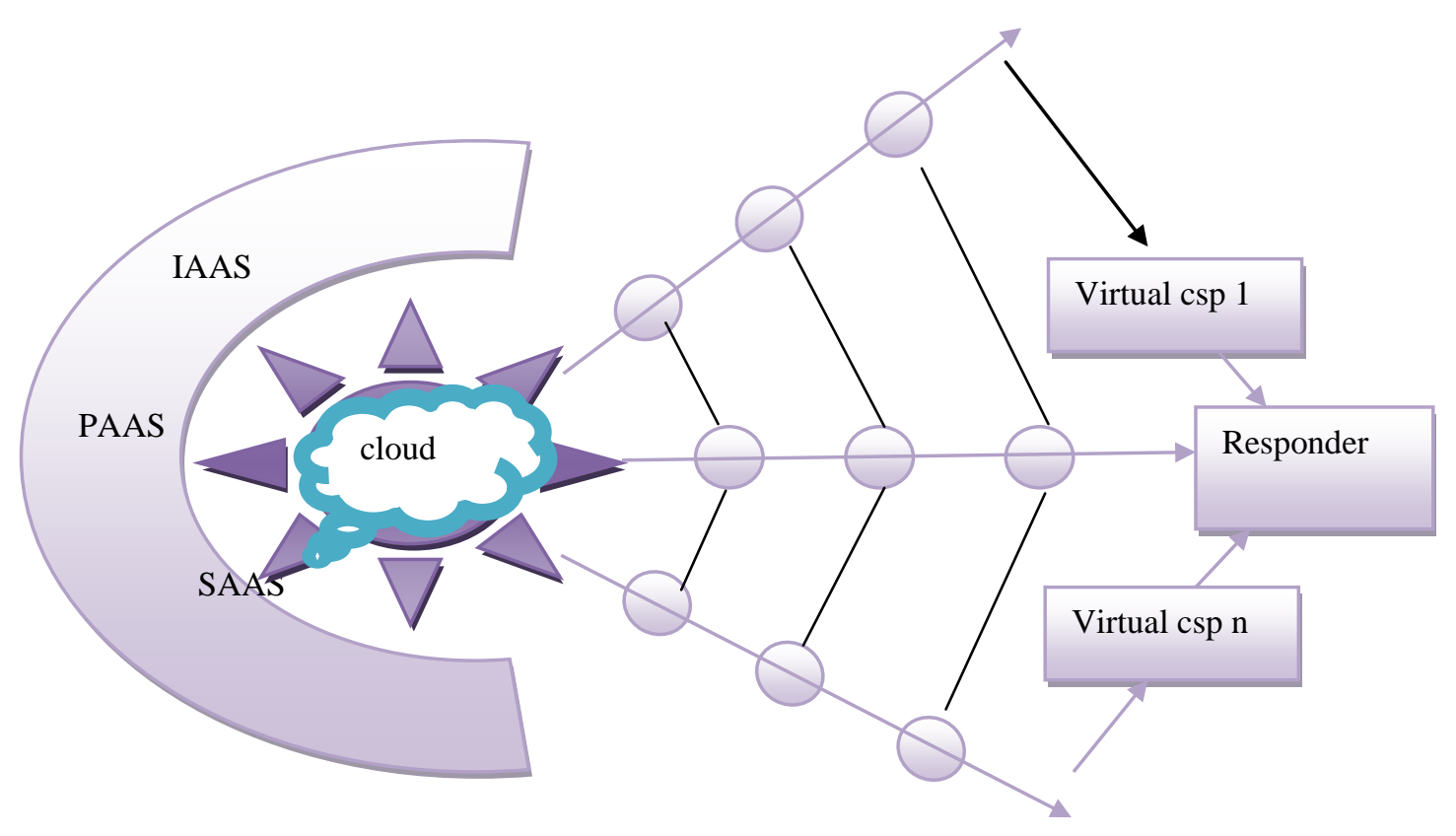

Figure 3.1: Cloud Service Provider

The workload of a request are wrapped in the VMs may transform the resources dynamically. The resource providers of applications are shown in figure 3.1.The cloud service provider can be highly variable outstanding to the instance of the progression effect, an impulsive surge in user requests, and other reasons may delay as defect condition. Moreover, some applications such as on stipulate data handing to the responder and virtual desktop have the random workload. It is inflexible to forecast their resource sharing stress switching. Even though server consolidation has the consequence of absorbing work load fluctuation, the amount of the peak resource requires of VMs distribution a physical machine PM) can be a large amount higher than the mean estimated value. Thus, it is repeatedly incompetent to over condition property based on the hit the highest point demand because doing switching process. So it will depart the resources under the time utilized most of the mitigation leads. Survive relocation allows a VM to be stimulated from one server to extended VM without interrupt the request running inside main resource provider. This is capable of being worn to adjust the VM describe for load harmonizing or energy saving purpose the provide CSP to utilize the VM scheduler for higher preemptive scheduling when the standard server consumption in the system become besides low, the VMs can be cumulative to a fraction of the servers response.

\section{A. Meta-scheduling Virtual Consolidation for Resource Sharing in Green Cloud}

In virtual consolidation, scheduling facilitates information about the content and preemptive information called metascheduling has been developed. The cloud server systems typically share common resource goals (e.g. to hide mitigation of parallel processing or fault tolerance), they aim at different fields of application to be shared dynamically to reduce the request waiting time using $\mathrm{VM}$. The virtual machine is deliberate to run data analysis of maximum precedence packet with meta information on a large amount of resource data, which is expected to be the maximum resource that across an outsized set of a share, not anything accessed servers. Once a user has fit to transfer the program dynamically set into the required map and reduce pattern, the completing framework acquires care of access the packet into subtasks, distributing and executing them. A solitary Virtual machine packet at all times consists of a distinct map and allots to decrease response time. We focus on the initiated sub problem of host response the server overload detection. Detecting when a host becomes overloaded directly influences the quality of service QoS, because the resource are dynamically allotted to switch the resource capability is completely utilize, it is extremely shared to complete data response that the applications are knowledge resource deficiency and presentation of data degradation. It makes the trouble of host overload deadlock detection composite is the requirement to optimize the time-averaged activities of the system, while treatment a variety of assorted workloads positioned on a single host. To address this setback, most of the present approaches to dynamic VM consolidation be appropriate either heuristic-based techniques with the scheduling algorithm, such as standing utilization thresholds decision-making support on statistical examination of multi sharing data or simply the intermittent adaptation of the priority VM allocation. The restrictions of this requested approach are that they lead to suboptimal consequences and do not allow the administrator to unambiguously set a QoS goal to deliver data. In other words, the presentation regarding the QoS distribute by the system can only be familiar indirectly by tuning parameter of the applied host excess detection algorithm. In dissimilarity, our approach enables the multischeduling with a circular preemptive system to explicitly specify a QoS goal in requisites of a workload sovereign QoS metric. The underlying analytical model allows a derivation of an optimal randomized control policy for any known stationary workload and a given state configuration. 


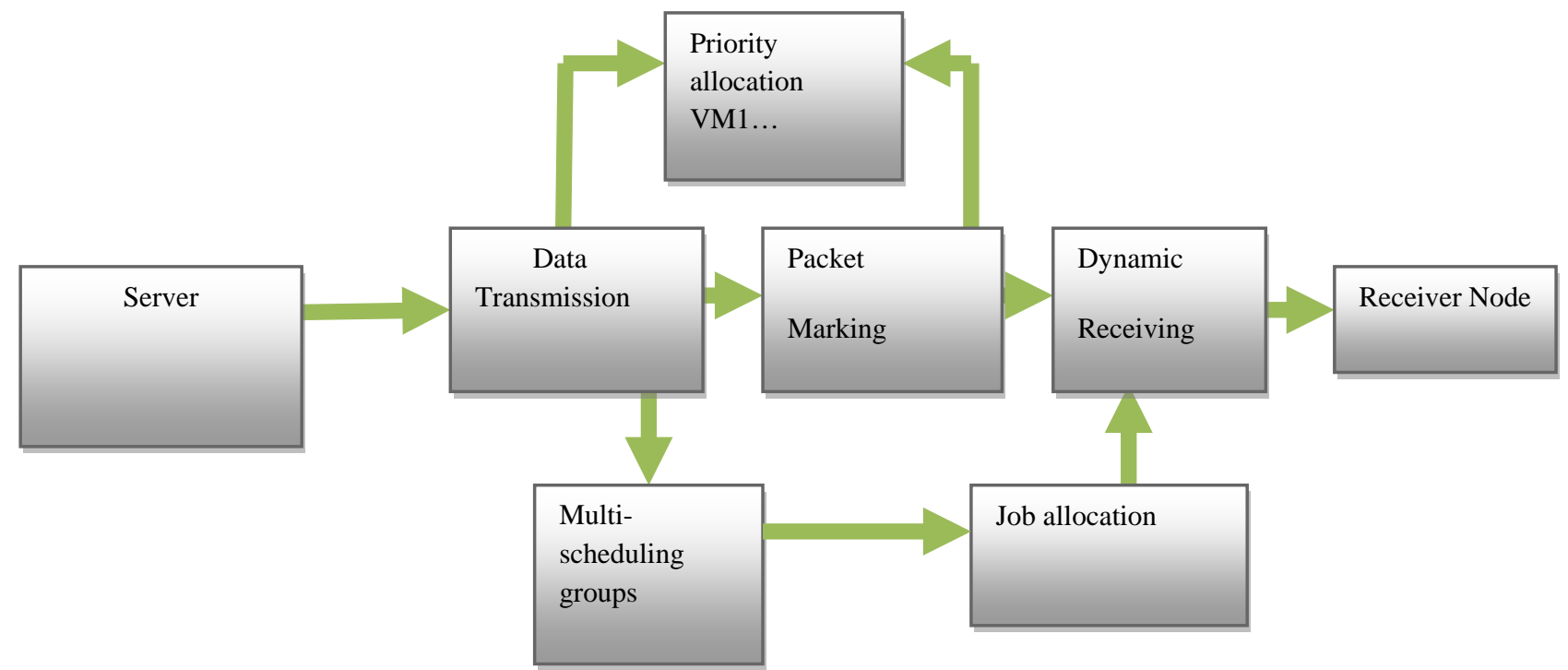

Figure 3.2: The Data Distribution Based on Virtual Machine in Cloud Data Centers

Figure 3.2 shows data distribution of VM, the workload be to check the task schedule property of resource data packets are allotted with data distribution the $\log$ trace $(\mathrm{Lt})$. Which implies resource hold less state transitions packet (p) and an exponential dynamic distribution of state transition delays are in multiple states. These work load assumption must be taken into account in an assessment of the applicability resource profile (wp) in data size. The proposed VM model to a shared system of payload (pl), with target server (ts).

\section{Algorithm}

step1: start

step2: initialize

step3: read data log trace Lt and Resource profile wp.

step4: receive a packet $\mathrm{P}$

Identify packet trustworthy $\mathrm{Pt}=\int_{1}^{N} \emptyset \times(W p(P$. Parent $))$

if true then

Compute pay load Pl.

Compute target server ts.

Compute target port tp.

If $\int t s, t p \in W p . R l$ then //ts and tp present in Red list Rl then

Switch packet and generate deliver log trace in lt.

$$
\mathrm{Tl}=\mathrm{Tl}+\mathrm{Tl}_{\mathrm{i}} .
$$

else

Generate Trace and forward packet.

$\mathrm{Tl}=\sum T l i+\mathrm{Tl}_{\mathrm{i}}$.

end.

else

switch response and generate deliver log trace in lt.

$$
\mathrm{Tl}=\sum T l i+\mathrm{Tl}_{\mathrm{i}} \text {. }
$$

end.

step 5: stop.

\section{B. Resource Allocation Packing Data}

The workload of applications resource is carried in the VMs change dynamically by a switch over resource process. The compares the demand services due to switching state Meta information process the revival comparison of delivered data response rate. Resource to that are allocate from the main server to virtual server, Moreover the target server ts, some pay load server on such VM server processed on demand service. To trace forecast, their resource demands with target port tp. Payload server (ps) consolidation to take the effect of balancing load variation, the amount of the max out resource demand of VMs distribution a physical machine (PM) can be a significant amount higher than the mean shared the resource by target system (ts).

\section{Remote Method Workload Analyzing}

For provisioning to be meaningful, the request process have led to workload has to exhibit some inevitability by remote switching for workload analyzing by maximum priority state. We presume the availability of a delegate workload, which can be attained either manually (e.g., by the resource provider or the administrators) or by design (through DBMS profilers). We require that the representative remote workload consists of a position of query classes all along with the predictable distribution of request across the dissimilar state. In either case, the stretch of cloud resources allows quick re-provisioning using VM's.

\section{3.4 Scheduling the server to response}

The approach presented periodically performs an intent scheduling resource bin packing model that to calculate a new VM layout, where the deadlock occurrence are eliminated, and the amount of requested servers in use is minimized. Scheduling response is then migrated accordingly. Scheduling server to an additional without interrupting the submission management inside. This can be used to adjust the VM present for workload balancing or energy saving to reduce the 
purpose. The virtual layout, however, might be quite different tasks from the single one, which may acquire a large numeral of VM migrations.

\section{E. VM Live Migration of Data}

This virtual server approach that uses live migration switching data to allocate data center resources dynamically based on request demands and hyper gear supports green computing by optimizing the quantity of servers used to reduce cost and power. To expand a practical bin packing resource allocation VM scheduling algorithm. It is proficient of both burden avoidance and green computing management. It is appropriate to a situation with multi-dimensional resource constraints are intended. We give a hypothetical proof that the number of servers, where the virtual servers are the number of blockage resources. Particularly, with only one blockage resource, the number of requested servers in use is delimited by the optimal value. And also establish that the numeral of VM migrations is delimited as well sharing to widespread simulation and simulated experiments. The processed results display high performance and reduced resource utilization contrast with the job allocation.

\section{F. Workflow Data Response}

Cloud providers refer to realize resource sharing of requested data from an isolated place based on user requirements. Cloud connections are essential to access in sequence and utilize possessions. Increase in the challenge on how to convey and where to accumulate data and calculate data are the subject caused by huge distributed file classification in cloud computing. Cloud Virtual Technologies such as Map Reduce Standard, Extended Virtualization and Distributed heading Systems are used to accomplish scalability and reliability process in clouds.

The load interpreter calculates approximation the resource difficulty in the near VM's. The scheduling algorithm optimizes VM outline task that the resource difficulty are satisfied and resource desecrates is minimized. Although the VM scheduler might appear to be a solitary point of breakdown the task, it is not concerned in the data pathway during standard processing resource sharing. Otherwise, the algorithm carries out overload prevention by expedition away a quantity of the VMs on the congested PMs. The scheduling algorithm also combines VMs on the underutilized PMs so that a number of the PMs become inactive and can be position into the stand-in mode to accumulate energy. The harvest of the scheduling algorithm is a VM relocation list which is then transmits to the $\mathrm{VM}$ on the concerned PMs for implementation. We presuppose that all PMs share backside process storage for resource sharing.

\section{RESUlt AND DisCUSSION}

The performance of the meta-scheduling projected algorithms is estimated in this section. The experiments were performed on a $2.80 \mathrm{GHz}$ Intel Core 2 Duo Processor with 3.5 GB memory. The operating system is Microsoft Windows 7. The simulated that are implemented in visual studio framework c\# language. Both real and server client VM are used in the experiments. File process was generated from the data generator with responding time management. Real world Server extended, process are obtained from virtual Repository scheduling is optimized with the server is obtained from shared resource is acquired from scheduling.

Dynamic Data allocation is a significant task distribution be supposed to maximize transfer rates and minimize in sequence data redundancy. The VM's algorithm to reduce the burden on the virtual machine. Set of heuristics that prevent burden in the system effectively while saving energy used. The primary processing components a central data repository which has the files to be deployed onto all the VMs. The explicit to virtual server holds the number of VMs are twisted to share and started within the selected cloud provider like the primary machine (PM) as well as job scheduling process. This process of scheduling that performs the definite consumption of the virtual communications environment, setting up a resource, and arrangement of the data processed in the VMs in our explicit case it is allotted VMs and the peers to peers (p2p) for distributing the partitioned file share process. Automatic Deployment Layer using the configuration parameters taken from the user. The proposed dynamic data distribution on demand service in cloud datacenters using virtual resources approach has been implemented and tested for its efficiency and accuracy. The method has shaped good consequences on constraint accuracy and has produced well-organized results with less period complexity.

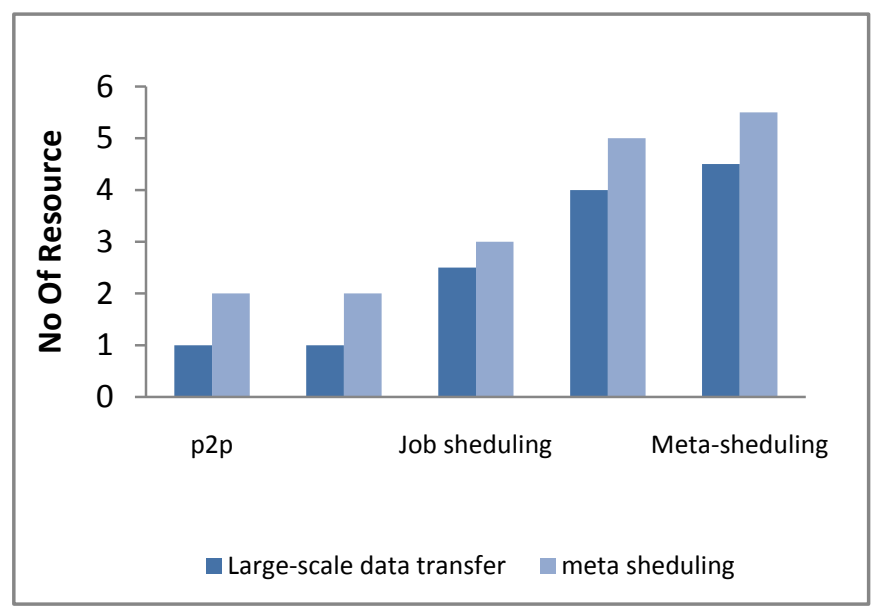

Figure 4.1: Data Transfer Rate in Extended Server

Figure 4.1 shows the various resource with server virtualization, the resource-based data transfer performance evaluation that divides the physical server into multiple virtual environments have a high-performance result. 


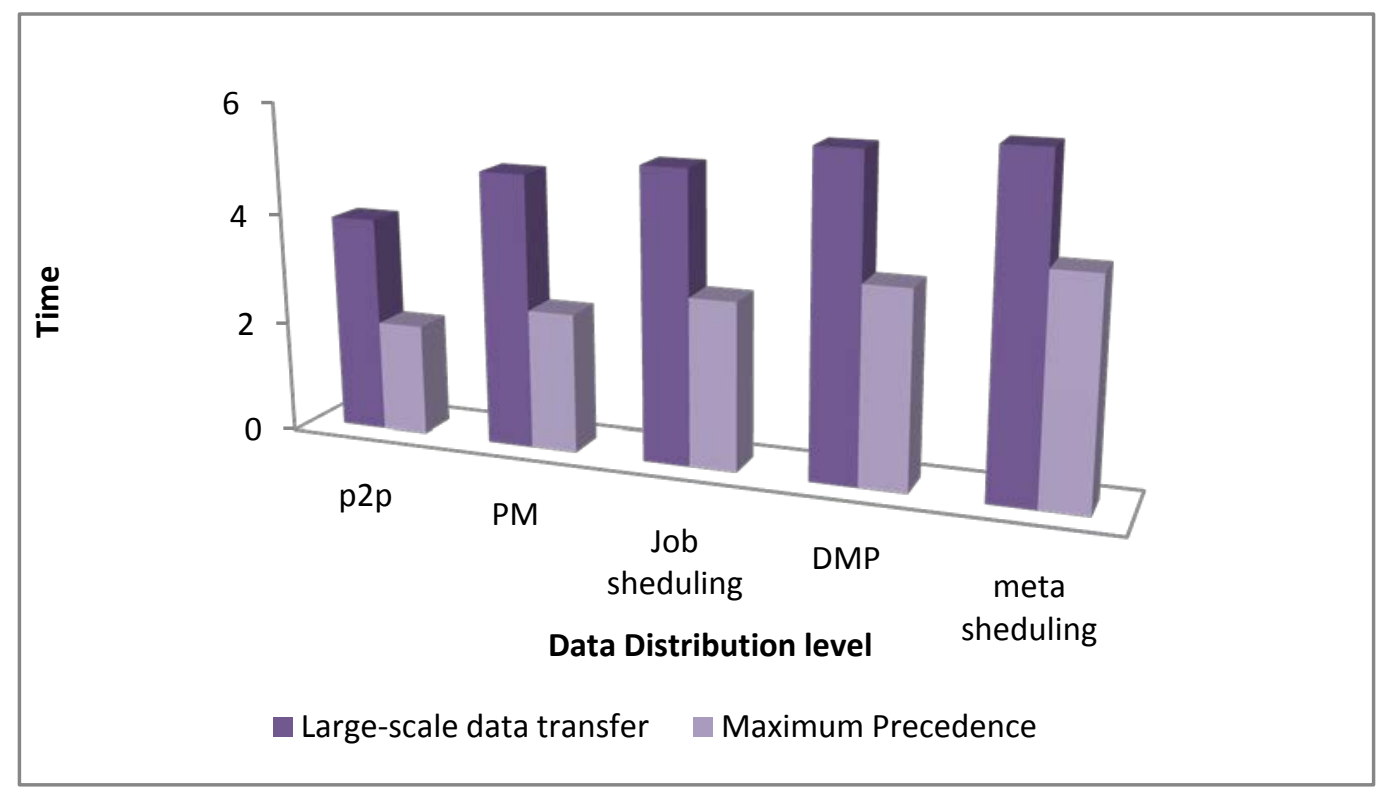

Figure 4.2: Data Distribution Level

Figure 4.2 shows the VMs , have time dependency comparison based on the priority The highest-priority of visualization queue in DMP and can preempt data in other queues based on a selection of cloud data providers Server virtualization is the improved time performance is smaller virtual servers to help maximize your server resources. The request run on virtual servers that are migrating to switch the assembler to configure using virtual machines, in addition to virtual intent servers is point onto each substantial server in the system to respond in scheduling queue.

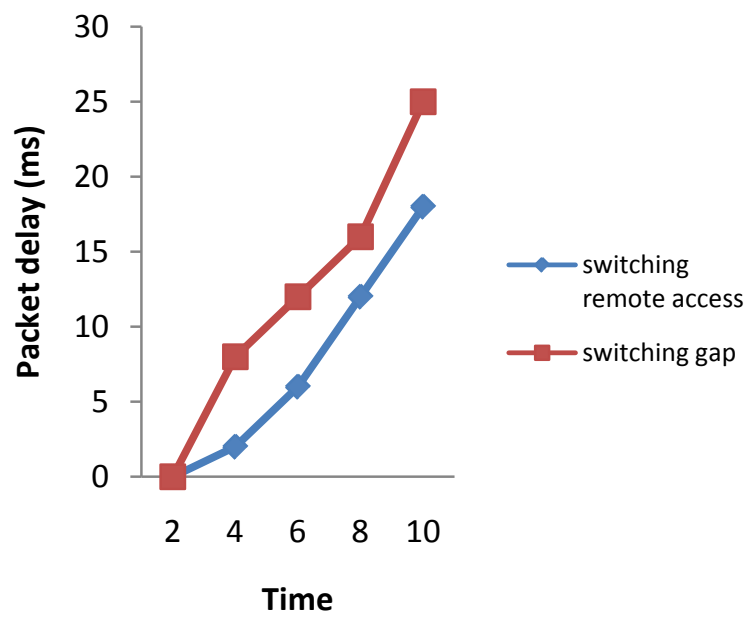

Figure 4.3: Time Distribution

Figure 4.3 shows distributed time estimation of Machine aware distributed resource scheduler (DRS) is a tool that balances and distributes computing volume by using available resources in a virtualized environment. They using dynamic waiting migration response time all of the resource sharing systems can accomplish system load stability, but common dynamic passage task would employ a great number of resources sharing that capacity leads to demeaning the entire system performance of task allocation.

\section{CONCLUSION}

To conclude multi-scheduling ensures minimum end-toend data transmission for the highest priority data while exhibiting acceptable fairness towards lowest-priority data. Experimental results show that the proposed meta-scheduling scheme has better performance than the existing and MultiQueue Scheduler shared regarding the minimum job behind deliver time and end with migrating-delay. As enhancements to the projected meta schedule scheme, we predict transfer task priority based on data's target that is instead of the straight task processing in shortest time. To decrease handing out job scheduling operating cost and save bandwidth, and also reflect on removing tasks with expire time limit from the intermediate task. Furthermore, if a real-time task seizes the resources for a much more period, additional tasks require waiting for an indeterminate period, reason the happening of a deadlock. This deadlock state of affairs degrades the routine of task scheduling system regarding the end to finish delay. Hence, the virtual machine would contract with the circular wait and preemptive circumstances to avoid deadlock from multi-task scheduling and also validate the simulation result and produce high performance using a real test-bed.

\section{REFERENCES}

[1] X. Shu and D. Yao, Data leak detection as a service, 2012.

[2] K. Borders and A. Prakash, "Quantifying information leaks in outbound web traffic", IEEE Symposium on Security and Privacy, Pp. 129-140, 2009.

[3] H. Yin, D. Song, M. Egele, C. Kruegel and E. Kirda, "Panorama: capturing system-wide information flow for malware detection and analysis”, Proceedings of the 14th ACM conference on Computer and communications security, Pp. 116-127, 2007.

[4] K. Li, Z. Zhong and L. Ramaswamy, "Privacy-aware collaborative spam filtering", IEEE Transactions on Parallel and Distributed systems, Vol. 20, No. 5, Pp.725-739, 2009.

[5] M. Cai, Y.K. Kwok, K. Hwang, S. Song and Y. Chen, "Collaborative Internet worm containment”, IEEE Security Privacy, 2005.

[6] U. Kang, C.E. Tsourakakis and C. Faloutsos, "Pegasus: A petascale graph mining system implementation and observations”, Ninth IEEE International Conference on Data Mining, Pp. 229-238, 2009. 
[7] B. Shao, H. Wang and Y. Xiao, "Managing and mining large graphs: systems and implementations", Proceedings of the 2012 ACM SIGMOD International Conference on Management of Data, Pp. 589-592, 2012.

[8] L.M. Vaquero, F. Cuadrado and M. Ripeanu, "Systems for near realtime analysis of large-scale dynamic graphs", arXiv preprint arXiv:1410.1903, 2014.

[9] L.G. Valiant, “A bridging model for parallel computation”, Commun. ACM, Vol. 33, No. 8, 2011.

[10] K. Shvachko, H. Kuang, S. Radia and R. Chandler, "The Hadoop distributed file system", 26th symposium on mass storage systems and technologies (MSST), Pp. 1-10, 2010. 